\title{
Conditional emotional response with humans: The effect of a variable interstimulus interval using a trace conditioning paradigm
}

\author{
DAVID A. SACHS AND JACK G. MAY, JR.
} FLORIDA STATE UNIVERSITY

\begin{abstract}
A variable interstimulus interval was used in conjunction with a trace conditioning paradigm in an attempt to maximize "anxiety" by increasing the uncertainty of the occurrence of a noxious stimulus, and thus increasing the probability of obtaining conditioned suppression with humans. No evidence of suppression was obtained nor was there any differential responding to low, medium or high frequency levels of CS-US pairings.
\end{abstract}

In 1941, Estes and Skinner demonstrated that ongoing behavior could be interrupted by the presence of a CS that had been paired with a noxious UCS. This interruption of the ongoing behavior has been referred to as "conditioned suppression" or "conditioned emotional response (CER)." The importance of the conditioned suppression procedure for investigating problems of "anxiety." "emotion," or "stress" is that these constructs are operationally defined in terms of the antecedent operations, and consequent behavioral effects as such are independent of physiological measures.

Although the literature is almost void in respect to humans demonstrating the CER, ample evidence for this behavioral effect has been demonstrated in primates (Brady \& Conrad, 1960). Kanfer (1958) used human Ss in a CER paradigm and reported that the behavioral measure, verbal rate, increased with CS onset. Edelman (1965) reported that of five Ss who performed on an eight stimulus matching task, two demonstrated response facilitation, one showed response suppression, and two showed no change from their performance baseline.

The purpose of the present study was to examine the behavioral effect of a CER paradigm designed to maximize the uncertainty of the occurrence of the noxious stimulus. The use of a trace conditioning paradigm with a variable interstimulus interval is designed to maximize the Ss' anxiety, since the cue which indicates the approximate onset of the US will be removed. When the interstimulus interval is constant, this provides a temporal cue to the onset of the US and with a delayed conditioning paradigm, the presence of the CS provides a physical cue which accompanies the onset of the US. Subjects

Ss were three male and one female student in attendance at Florida State University.

\section{Apparatus}

The manipulandum consisted of a lever switch mounted on a 5-1/2 $\times 4-6 / 8 \times 5-1 / 4$ in. wooden box. A counter, which indicated the number of points the $S$ received, was mounted directly above the lever switch.
The various frequencies of the $\operatorname{CS}(1 \mathrm{~K}, 2 \mathrm{~K}$, and $3 \mathrm{~K})$ were produced by a General Radio Co. oscillator and were delivered via the speaker of a Webcor tape recorder, which was located in the room with the S. The US was capacitor shock, produced by a Variac and converted to dc by a rectifier. The timing of pre- and post CS periods, the operation of the respective pre- and post CS counters, and the presentation of CS and US were automatically performed by the use of electromechanical counters.

Procedure

Ss were seated in a room adjacent to the room containing the programming equipment. Sessions were $45 \mathrm{~min}$, during which time Ss were instructed to press the lever to receive points. The points were presented, one per reinforcement, on a VI 30 sec schedule. After the S's rate of lever pressing had stabilized for several sessions, and prior to the initiation of suppression training, finger tip electrodes were placed on the second and third fingers of the inoperative hand. At this time, Ss were tested for pseudoconditioning.

\section{CER Training}

Immediately prior to the first CER session, Ss were given the following instructions: "While you are working you will hear a tone come on for a brief period of time. The tone you hear is a signal that shock will occur sometime after the tone goes off. The frequency of the tone will give you some idea of the intensity of the shock that will occur."

Following the instructions, shock intensity levels were determined for the three following conditions: (1) Low -when the S first detected the shock; (2) Mediumwhen the shock was reported as being "uncomfortable"; (3) High-when the shock was reported as being painful. With respect to the "painful"' level, the obtained Variac setting was increased by $5 \mathrm{~V}$ to maximize the possibility that this intensity level was aversive. The range of intensity levels used were: Low, 28-35 V dc; Medium, 70-80 V dc; High, 110-120 V dc.

Each CS was always paired with the same shock intensity; thus the $3 \mathrm{~K}$ CS was always paired with high intensity, the $2 \mathrm{~K} \mathrm{CS}$ with the medium US intensity, and the low CS with the low US intensity. Each of the three CS frequencies was presented four times per session, the duration of each CS presentation being 3 sec. The US followed the CS on a variable interstimulus interval $(10,20,30$, and $40 \mathrm{sec})$, with the average length of the interstimulus interval being $25 \mathrm{sec}$. The order of presentation of both the US and the interstimulus interval 


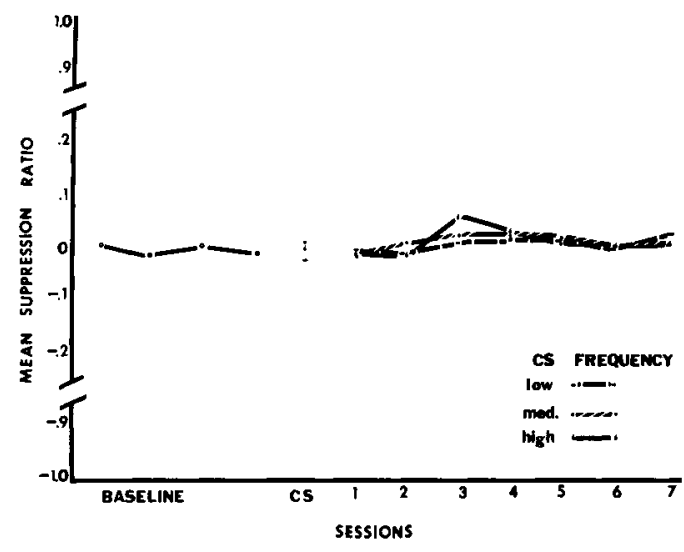

Fig. 1. Mean suppression ratios for $S F$ under conditions of baseline, pseudoconditioning, and CS-US pairings for each of the three frequencyintensity levels. This data is representative of that produced by the other three Ss.

was determined by using a table of random numbers. A different randomized series was used for each session. Resulis

The criteria for determining the effect of the CS-US pairing on the rate of responding was the suppression ratio proposed by Dinc (1965). This ratio is $A-B / A+B$, where $B$ represents the number of responses between CS onset and US onset, and A represents the number of responses prior to $\mathrm{CS}$ onset for an interval equal to the $\mathrm{B}$ interval.

The range of suppression ratios obtained during suppression training, independent of the CS-US level, was: -.015 to .062 for $\mathrm{F}$; -.037 to .019 for $\mathrm{G} ;-.048$ to .032 for $\mathrm{J}$; and -.025 to .030 for $M$. These ratios represent a difference of one to three responses between pre- and post CS intervals. Thus there was no suppression noted at any time during the experiment. In addition to the absence of suppression, no differential effect was observed in the rate of responding to the three different CSs. Figure 1, which illustrates the results obtained from $F$, is typical of the results obtained from the other three Ss.

It was observed that the high intensity US tended to produce a decrease in the overall response rate within a given session, although this decrease was less than $10 \%$ of the overall rate. Thus, for example, F emitted approximately 56 responses/20 sec in the early part of the session, but following several high intensity US presentations, his rate would decrease to approximately 51 responses $/ 20 \mathrm{sec}$.

Discussion

The failure to obtain conditioned suppression with humans is partially in agreement with Kanfer (1958). What is surprising is that, whereas Kanfer obtained an increase in responding, the results obtained in this experiment falled to indicate either a systematic increase or decrease in responding.
Although quantitatively no evidence exists that the CER was obtained, several Ss repeatedly verbalized their anxiety at the conclusion of a session. Among the comments made by the Ss were: "When the strong tone comes on I bite my lip"'; 'I brace myself to get ready for the shock"; "I started to try to figure out when the shock was coming so I could get ready for it but it didn ${ }^{\mathrm{t}} \mathrm{t}$ always come on at the same time." These verbalizations tend (qualitatively) to support the initial belief that the use of a trace conditioning paradigm with a variable interstimulus interval would tend to maximize anxiety in the sense of maximization of anxiety being defined as the "uncertainty of the occurrence of a noxious stimulus."

If verbal reports of anxiety are accepted, the basic question becomes: "Why do humans fail to show a performance decrement during periods of experimentally induced anxiety?"

The first possible explanation is that human beings, and college students especially, are used to working under varying degrees of what may be termed "anxiety." "Biting one's lip" and "bracing oneself" are learned behaviors which may enable the individual to somehow reduce the effect of the noxious stimulus while continuing to emit the desired response.

A second possible explanation is that social reinforcement by the $\mathrm{E}$ is powerful enough to outweigh the anxiety produced by the CS-US pairing. The Ss tended to develop the set that they were to continue to press the bar and, as one $S$ indicated upon termination of the experiment, "I thought you wanted me to keep pressing no matter what, so that's what I did."

A third posstble explanation is that the effect of the CS-US pairing on response rate may be a function of the type of response required. Whereas Kanfer's verbal response increased due to CS-US palring, the lever pressing response for points failed to show any systematic change. The possibility exists that with more complex responses which require careful and sustained attention on the part of the S, CS-US pairing may produce a response decrement.

\section{References}

BRADY, J. V., \& CONRAD, D. G. Some effects of limbic-system selfstimulation upon conditioned emotional behavior. J. comp. physiol. Psychol, 1960, 53, 128-137.

DINC, E. H. The olfactory system in the detection of ionizing radiation. Unpublished Ph.D. dissertation, Florida State University, 1965.

EDELMAN, R. Application of the conditioned suppression paradigm to human subjects. Unpublished M.A. thesis, Florida State University, 1965.

ESTES, E. K., \& SKINNER, B. F. Some quantitative properties of anxiety. J. exp. Psychol, 1941, 390-400.

KANFER, R. H. Effect of a warning signal preceding a noxious stimulus on verbal rate and heart rate. J. exp. Psychoh, 1958, 55, 73-79.

Note

1. The author wishes to express his appreciation to Dr. Baron B. Scarborough, who provided the laboratory facilities for this study. 